\title{
Estimation of global left ventricular function: a comparison between echocardiography and cardiac magnetic resonanace
} MC D Torres

\author{
Address: National university hospital, singapore, Singapore \\ from I3th Annual SCMR Scientific Sessions \\ Phoenix, AZ, USA. 2I-24 January 2010 \\ Published: 21 January 2010 \\ Journal of Cardiovascular Magnetic Resonance 2010, I2(SuppI I):PI78 doi:I0.I I86/I532-429X-I2-SI-PI78
}

This abstract is available from: http://jcmr-online.com/content/I2/SI/PI78

(C) 2010 Torres; licensee BioMed Central Ltd.

\section{Introduction}

Estimation of global left ventricular (LV) function is important in the evaluation of patients with cardiovascular disease. Prognosis of patients is largely dependent on LV function. 2D echocardiography (2DE) and cardiovascular magnetic resonance (CMR) have been used increasingly as the imaging modalities of choice for noninvasive assessment of LV function.

\section{Purpose}

This study sought to compare both M-mode and visual assessment of left ventricular ejection fraction (LVEF) from 2DE with the "gold standard" technique, CMR.

\section{Methods}

One hundred consecutive adult patients with stable cardiovascular disease underwent 2DE and CMR (Siemens, 1.5 $\mathrm{T}$ ) within one week apart. LVEF measurements using $\mathrm{M}$ mode echocardiography (M-mode $\mathrm{EF})$, visual estimation on 2DE (visual EF), and CMR volumetric analysis (CMR $\mathrm{EF})$ were retrospectively reviewed in 58 patients with normal LVEF (CMR EF $>/=50 \%), 17$ patients with moderate LV dysfunction (CMR EF 35-49\%), and 25 patients with severe LV dysfunction (CMR EF < 35\%). Experienced sonographers quantified the $\mathrm{M}$-mode $\mathrm{EF}$ in all studies according to standard Teichholz method. Level 3-accredited echocardiologists and CMR readers blindly assessed LVEF visually on echocardiograms and performed off-line CMR LV volume analysis, respectively. CMR served as the gold standard for comparison with M-mode EF and visual EF. Statistical analysis included Pearson correlation coefficient and paired samples $\mathrm{T}$ test.

\section{Results}

Correlation between visual EF and CMR EF was fairly good $(\mathrm{r}=0.55)$ but was poor between M-mode EF and
CMR EF $(r=0.36)$. In comparison with CMR, visual EF showed moderate correlation in patients with normal EF $(r=0.48)$, moderate LV dysfunction $(r=0.58)$, and severe $\mathrm{LV}$ dysfunction $(\mathrm{r}=0.61)$. M-mode measurements correlated less well with CMR in patients with both moderate $(\mathrm{r}=0.28)$ and severe $(\mathrm{r}=0.24) \mathrm{LV}$ dysfunction. However, M-mode measurements had closer agreement with CMR in patients with normal EF $(\mathrm{r}=0.57)$. Overall, there was a satisfactory correlation of 2DE and CMR LVEF's.

\section{Conclusion}

In our laboratory, echocardiography correlates acceptably well with CMR in global LV function. In patients with LV dysfunction, visual EF showed better agreement with CMR than M-mode EF. Though less sophisticated than high-resolution CMR technique, visual EF provides a fairly accurate means of estimating global LV systolic function for routine use in clinical practice. 